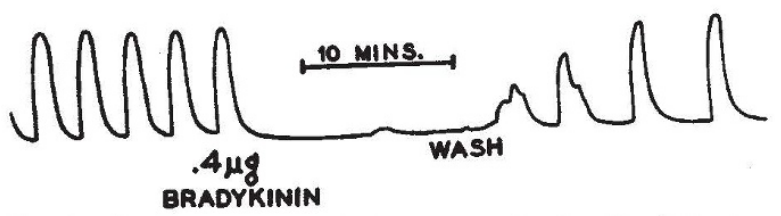

Fig. 1. In the non-pregnant uterus prompt relaxation following bradykinin with recovery is demonstrated

least one of these variables was considered a relaxant response. In the non-pregnant uterus a concentration of $0.2 \mu \mathrm{g} / \mathrm{ml}$. produced a significant relaxant response in $\mathbf{5 7}$ per cent of the tests. At levels of $0.8 \mu \mathrm{g} / \mathrm{ml}$. a maximum response in the non-pregnant uterus resulted in a complete inhibition of all contractions. In the pregnant uterus, as indicated in Table 1, slightly higher concentrations were required to inhibit contractility. Oxytocininduced contractions required slightly higher levels of bradykinin than the spontaneous variety. This was particularly true in the pregnant muscle. Fig. 1 shows a low concentration of bradykinin-inhibiting contractions of the non-pregnant uterus in the presence of oxytocin and a return to the original pattern following a wash.

The high potency of this compound on human uterine muscle with its inhibiting action on contractions is of considerable interest. A study of bradykinin on in situ human uterus is indicated to determine its possible effect in preventing the onset of premature labour. The known hypotensive effect of bradykinin makes it unlikely that its use alone may be practical. A combination of a hypertensive agent, such as angiotensin, with bradykinin may provide effective therapeutic agent.

ROBERT LANDESMaN

Wimliam L. Campbell

KATHLEEN WILSON

Cornell University Medical College,

Department of Obstetrics and Gynecology, 530 East 70th Street,

New York 21.

1 Boissonnas, R. A., Guttmann, S. T., and Jaquenoud, P. A., Helv. Chim. Acta, 43, 1481 (1960).

${ }^{2}$ Elliot, D. F., Horton, E. W., and Lewis, G. P., J. Physiol., 153, 473 (1960).

- Berde, B.. and Saameli, K., Nature, 191, 83 (1961).

- Coutimho, E. M., and Csapo, A., J. Gen. Physiol., 43, 131 (1959).

\section{Effect of Hydrogen Ion on the Rates of Migration of Sodium and Potassium lons : Possible Relationship to Magnetism}

I HAVE read with interest the recent communication of Salminen ${ }^{1}$ on the effect of hydrogen ions on the differential rates of migration of sodium and potassium ions. I believe this may relate to an experiment which $I$ have performed demonstrating a differential effect of extermal magnetic fields on the concentration of these ions across a semipermeable membrane.

A 4-in. length of 'Cellophane' tube of the sort used in the artificial kidney apparatus was filled with the usual dialysate solution for blood lavage, the ends were sealed and it was suspended in a beaker of the same solution. The pole of a permanent magnet capable of lifting a 125-lb. weight of steel was placed against the 'Cellophane' tube so that the strongest magnetic field was inside the tube. After incubation for $9 \mathrm{~h}$ the concentration of ions in the two compartments was determined by standard methods available in the clinical laboratory. The experiment was repeated five times, and in each instance the results were similar to those given in Table 1 .

\begin{tabular}{lrccccc} 
& \multicolumn{7}{c}{ Table 1 } & & \\
'Cellophane' tube & $\mathrm{Mg}$ & $\mathrm{Ca}$ & $\mathrm{K}$ & $\mathrm{Na}$ & $\mathrm{Cl}$ & $\mathrm{HCO}_{3}$ \\
Beaker & $3 \cdot 5$ & $4 \cdot 5$ & $\mathbf{4} \cdot 9$ & 160 & 132 & $*$ \\
& $\mathbf{3} \cdot 5$ & $\mathbf{4} \cdot 5$ & $\mathbf{4} \cdot \mathbf{4}$ & $\mathbf{1 5 1}$ & $\mathbf{1 2 3}$ & \\
& All values in m.equiv./1. \\
& * Present but not determined.
\end{tabular}

The sodium, potassium and chloride ions were always more concentrated in the 'Cellophane' tube than in the surrounding bath. This difference could be increased greatly by decreasing the volume of solution inside the 'Cellophane' tube. The increase of potassium was always proportionately greater than the sodium. The failure to demonstrate any effect on magnesium and calcium may have been due to formation of unionized complexes with bicarbonate.

I have surmised that the movement of electrons along the chains of respiratory enzymes in the mitochondria and the concurrent movement of hydrogen ions in the same direction generate electromagnetic fields, as do other electric currents, and that these magnetic forces may be a determining factor in the characteristic proportions of intracellular ions, and thereby related to the mechanism of the 'salt pump'.

Department of Medicine,

University of Texas,

Galveston, Texas.

${ }^{1}$ Salminen, S., Nature, 195, 908 (1962).

Willitam K. George

Dr. GEoraE's communication contains no mention of control experiments without a magnetic field. Thus it is open to question whether the results, interesting as such, are related to some factors other than the magnet. The fact that the experiments were performed with dialysate fluid made for blood lavage suggests that its $P_{\mathrm{CO}_{3}}$ would have been higher than that of the air. The loss of carbon dioxido from the beaker might have produced a higher gradient of $\mathrm{H}^{+}$in the 'Cellophane' tube, resulting in an accumulation of other cations in this phase, as was found, for example, by Teorell in his classical work ${ }^{1}$. That the effect of $\mathrm{H}^{+}$on the rates of simultaneous non-equilibrium migration of $\mathrm{Na}^{+}$and $\mathrm{K}^{+}$is different was experimentally found in my communication referred to by Dr. George.

A theoretical treatment of these problems is in progress by our group, using the concepts of irreversible thermodynamics.

Wihuri Research Institute,

Stmo Salminen Helsinki.

1 Teorell, T., J. Gen. Physiol., 21, 107 (1937).

\section{Effect of 3:5:3'-triiodothyroacetic Acid on the in vitro Oxygen Uptake of Leuco- cytes of Hypopituitary Patients}

NeITHER L-3 : $5: 3^{\prime}$-triiodothyronine $\left(\mathrm{T}_{3}\right)$ nor $3: 5: 3^{\prime}$ triiodothyroacetic acid (TRIAC) affect the uptake of oxygen by leucocytes from normal individuals studied in vitro with the direct method of Warburg with a modified Krebs's No. 2 buffer. When, however, these in vitro investigations are carried out with leucocytes from patients with primary hypothyroidism, an immediate stimulation of oxygen uptake can be demonstrated with TRIAC although no effect is produced by $\mathrm{T}_{3}$ (ref. 1 ).

The opportunity was taken to carry out similar investi. gations on the leucocytes from 5 other patients who were also hypothyroid. In these patients, however, the hypothyroid state was the result of hypopituitarism and there was clinical and investigational evidence of the other expected endocrine deficiencies.

Blood was collected in heparinized siliconed tubes according to the method of Bisset and Alexander ${ }^{2}$. The leucocytes were isolated after sedimentation with dextran ( 6 per cent $\mathrm{w} / \mathrm{v}$ in 0.9 per cent sodium chloride solution). From 80-100 ml. of blood from each patient sufficient leucocytes were obtained to set up 4 flasks, each of which contained approximately 40-50 million leucocytes in Krebs's No. 2 buffer. TRIAC in two concentrations, 25 and 